11,13

\title{
Эффект формы при расслаивании твердых растворов в малом объеме на примере сплава Bi-Sb
}

\author{
(C) В.Б. Федосеев, А.В. Шишулин \\ Институт металлоорганической химии им. Г.А. Разуваева РАН, \\ Нижний Новгород, Россия \\ ๑ E-mail: Chichouline_Alex@live.ru
}

(Поступила в Редакцию 22 января 2018 г.)

\begin{abstract}
Описаны закономерности влияния формы межфазных границ в системах малого объема на расслаивание растворов с верхней критической температурой растворения (ВКТР) на примере частиц сплава $\mathrm{Bi}-\mathrm{Sb} \mathrm{c}$ конфигурацией core-shell. Изменение формы межфазных границ в общем виде моделируется введением параметра, соответствующего степени отклонения формы границ от сферической. Анализ экстремумов функции Гиббса выявил закономерности влияния формы core- и shell-фаз на фазовые равновесия, термодинамическую устойчивость гетерогенных состояний и диаграмму расслаивания. Показано, что отклонение формы межфазных границ от сферической меняет ВКТР и взаимную растворимость компонентов. Деформация оболочки core-shell частицы увеличивает термодинамическую стабильность гетерогенного состояния, что способствует распаду раствора. Деформация ядра понижает термодинамическую стабильность гетерогенного состояния и расширяет область метастабильных состояний.
\end{abstract}

Работа В.Б. Федовеева выполнена при поддержке РНФ (проект № 15-13-00137-П). Работа А.В. Шишулина выполнена при поддержке РФФИ (проект № 18-08-01356), РФФИ и Правительства Нижегородской области (проект № 18-43-520037).

DOI: 10.21883/FTT.2018.07.46128.013

\section{1. Введение}

Физические и химические свойства малых частиц и фазовые превращения в них давно вызывают интерес исследователей [1]. Потребность в этих исследованиях возрастает с развитием аддитивных технологий и расширением области применения наноматериалов. Особенности фазовых превращений в наноразмерных системах проявляются в смещении характеристических линий и точек на фазовых диаграммах по сравнению с диаграммами для макросистем, причем величина смещения может превышать сотни градусов и зависит от объема системы [2-5]. Поэтому при описании процессов и свойств наноматериалов справочные данные о диаграммах состояния требуют существенного уточнения с учетом размерных эффектов.

Описание фазовых превращений в наноматериалах сильно усложняется учетом эффекта формы [6-8]. Эффект заключается в том, что при постоянном объеме и химическом составе фазовая диаграмма зависит от формы системы и формы внутренних границ раздела фаз. Как отмечено в [9], этот аспект редко учитывается при моделировании наночастиц. Наиболее часто рассматриваются простые конфигурации типа core-shell со сферической $[10,11]$ и несферической (куб в сфеpe) [12] симметрией, есть расчеты для наночастиц с аксиальной симметрией со сферической (,janus“) [11] или плоской $[3,6,13]$ межфазной границей, для выпуклых многогранников $[14,15]$.

В этих работах не рассмотрено одно из важных проявлений эффекта формы, связанное с существованием и устойчивостью метастабильных состояний и фаз. Для core-shell систем малого объема одним из следствий эффекта формы является расщепление фазовой диаграммы на гетерогенные состояния, имеющие разные диаграммы расслаивания [16]. Эти состояния имеют различную термодинамическую устойчивость, области существования, состав фаз. Размерные эффекты и эффекты формы в подобных системах могут сопровождаться появлением, инверсией и исчезновением метастабильных фаз [17,18].

Термодинамический подход к построению фазовых диаграмм для систем малого объема реализован в методах CALPHAD [19,20]. Особенности и метастабильные состояния позволяет выявить дополнительный анализ экстремумов функции Гиббса [16-18].

Эффект формы имеет общую природу с другими размерными эффектами, природа которых связана с высокой долей поверхностной энергии. При этом любое изохорическое изменение формы, сопровождающееся изменением площади межфазных поверхностей, влияет на энергию системы. Это меняет положения и глубину экстремумов функции Гиббса и существенно смещает фазовые равновесия. В результате без указания геометрических характеристик оценка фазового состава неточна или некорректна.

При рассмотрении эффекта формы одной из задач является выбор геометрических конфигураций. Простые конфигурации удобны для моделирования, однако реальные системы чаще демонстрируют более сложную морфологию. Например, преобразования капель углеводородов микронных размеров, диспергированных в 
растворах поверхностно-активных веществ, при охлаждении образуют более десятка различных воспроизводимых конфигураций [21]. Большой обзор конфигураций структуры наночастиц приведен в [22]. Нельзя признать эффективным подход, основанный на моделировании конкретных наблюдаемых или переборе множества возможных конфигураций. Поэтому с целью обобщения результатов в работе использовано параметрическое задание формы. При этом возможность непрерывного изменения формы дают преобразования типа сфера сфероид, куб - параллелепипед. Отношения диаметров эллипсоида, сторон параллелепипеда являются параметрами, которые при заданном объеме определяют площади поверхностей этих фигур. Эти представления можно привести к еще более общему описанию, если при параметризации использовать отношение площади поверхности системы данной формы к площади поверхности сферы равного объема. Применяются и другие способы параметризации формы, например в виде отношения числа атомов на разных ребрах параллелепипеда [23].

Далее ограничим рассматриваемые состояния конфигурациями core-shell c несферической оболочкой или ядром. Форма shell-фазы может быть несферической в случае, если она задана стенками твердой матрицы, границами зерен, либо возникает в процессах диспергирования и деформации. Форма core-фазы может быть несферической из-за анизотропии свойств core-фазы или поля упругих напряжений, создаваемого внешними и внутренними факторами.

\section{2. Термодинамическое описание влияния формы системы на фазовые равновесия в системах малого объема}

В качестве объекта моделирования рассмотрим частицы расслаивающегося твердого раствора для случая, когда форма core- или shell-фазы отличается от сферической.

Для закрытой двухкомпонентной системы условия сохранения при фазовом превращении имеют вид

$$
\begin{gathered}
n_{2}=x n, \quad n_{1}=n-n_{2}, \quad n_{i c}=\theta_{i} n_{i}, \quad n_{i s}=\left(1-\theta_{i}\right) n_{i}, \\
x=\frac{n_{2}}{n_{1}+n_{2}}, \quad x_{f}=\frac{n_{2 f}}{n_{1 f}+n_{2 f}}, \quad \theta_{i}=\frac{n_{i c}}{n_{i c}+n_{i s}}, \\
f=c, s, \quad i=1,2,
\end{gathered}
$$

где $n$ - суммарное число молей, $n_{i}$ - число молей компонента $i, x$ - концентрация смеси, $n_{i f}, x_{f}-$ число молей и мольная доля компонента $i$ в фазе $f$, индексами $c$ и $s$ обозначены core- и shell-фазы соответственно. Величины $\theta_{i}$ соответствуют доле компонента $i$, перешедшего в core-фазу, Так как $0 \leq \theta_{i} \leq 1$, эти величины удобны для замены переменных и при сопоставлении результатов моделирования для разных состояний системы.

Уравнения сохранения (1) позволяют вычислить объем системы $V$ и сосушествующих фаз $V_{f}$ :

$$
V=n_{1} V_{1}+n_{2} V_{2}, \quad V_{f}=n_{1} V_{1}+n_{2} V_{2},
$$

здесь $V_{i}$ - мольные объемы компонентов.

Поверхностная энергия зависит от формы системы и межфазных границ. Минимальную площадь поверхности имеет конфигурация со сферической симметрией. В этом случае площадь поверхности системы $A_{s}$ и межфазной поверхности $A_{c}$ вычисляются из (2) как

$$
\begin{aligned}
& A_{s}=4 \pi r_{s}^{2}, \quad r_{s}=\left(\frac{3}{4 \pi} V\right)^{1 / 3}, \\
& A_{c}=4 \pi r_{c}^{2}, \quad r_{c}=\left(\frac{3}{4 \pi} V_{c}\right)^{1 / 3},
\end{aligned}
$$

где $r_{s}$ и $r_{c}$ - радиусы сфер объемом $V$ и $V_{c}$ соответственно.

Энергия Гиббса системы с учетом поверхностной энергии имеет вид

$$
g=\left(n_{1 c}+n_{2 c}\right) G\left(x_{c}, T\right)+\left(n_{1 s}+n_{2 s}\right) G\left(x_{s}, T\right)+g_{s u r f},
$$

где $x_{c}$ и $x_{s}-$ мольная доля компонента 2 в coreи shell-фазах, $G(x, T)$ - функция Гиббса раствора с концентрацией $x$ при температуре $T, g_{s u r f}-$ поверхностная энергия, которая содержит вклады внешней и внутренней межфазных границ

$$
g_{\text {surf }}=\sigma_{s} A_{s}+\sigma_{c s} A_{c},
$$

где $\sigma_{s}, \sigma_{c s}-$ поверхностная энергия твердого раствора на внешней (shell) и внутренней (core-shell) границах.

Для отображения состояния системы удобно использовать безразмерные переменные $\theta_{1}, \theta_{2}$, введенные выше (1), и избыточную по отношению к гомогенному состоянию функцию в пересчете на моль смеси

$$
G\left(\theta_{1}, \theta_{2}\right)=\frac{g\left(\theta_{1}, \theta_{2}\right)-g(0,0)}{n_{1}+n_{2}} .
$$

При постоянном объеме системы величины $A_{s}$ и $A_{c}$ определяются формой соответствующих границ. Для сферической поверхности $A_{s}$ и $A_{c}$ минимальны. Любая изохорическая деформация core- или shell- фаз увеличивает площадь границ, поэтому отклонение от сферической формы можно использовать в качестве параметра. Введем коэффициент формы $k_{f}=A_{f} / A_{f 0}$, равный отношению площадей поверхности рассматриваемой фигуры $A_{f}$ и сферы того же объема $A_{f 0}$. Например, для тетраэдра $-k_{f}=1.49$, куба $-k_{f}=1.24$, октаэдра $-k_{f}=1.18$. Для правильных многогранников с увеличением числа граней $k_{f} \rightarrow 1$, поэтому различия между результатами расчета для многогранников 

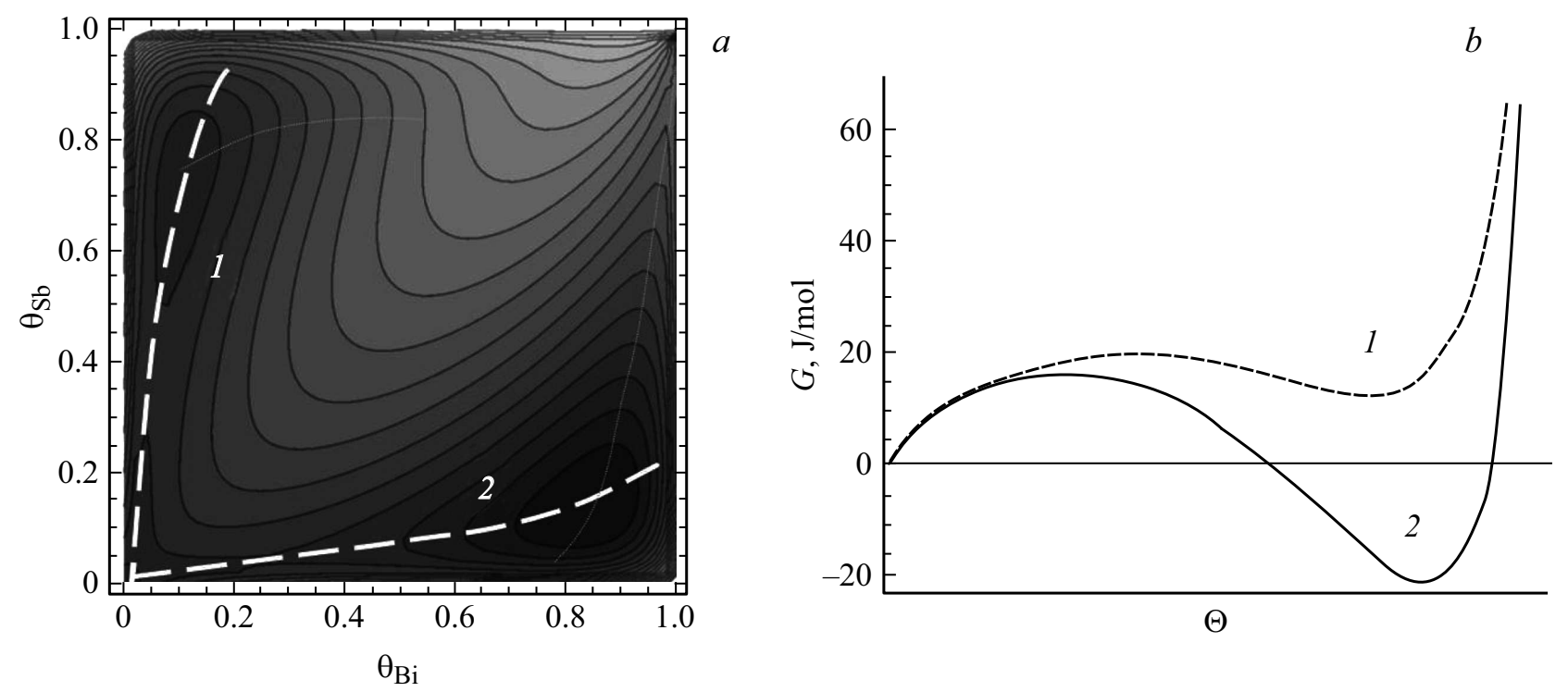

$\Theta$

Рис. 1. $a-$ - функция Гиббса сферической core-shell частицы радиусом $R_{0}=200 \mathrm{~nm}(T=280 \mathrm{~K}, x=0.5)$. Минимумам соответствует темная окраска. Траектории 1 и 2 проходят через $G(0,0)$ и минимумы функции в соответствии с условиями $\left(\partial G / \partial \theta_{i}\right)_{\theta_{j}}=0 . b-$ изменение функции Гиббса вдоль траектории 1 и 2.

и сферических наночастиц сглаживаются. Другим рассмотренным в литературе конфигурациям также можно сопоставить численное значение $k_{f}$. Это облегчает пересчет и сопоставление фазовых равновесий для систем с разной формой. Коэффициент формы является непрерывным параметром. Это позволяет обобщить разные варианты изохорических преобразований системы, включая плавные деформации. Этой же цели можно достичь, если использовать фрактальную размерность [24].

Учет формы системы при моделировании фазовых равновесий сводится к добавлению множителей $k_{f}$ в уравнение (5)

$$
A_{s}\left(k_{s}\right)=k_{s} A_{s}, \quad A_{c}\left(k_{c}\right)=k_{c} A_{c} .
$$

Это не требует существенной модификации расчетных моделей CALPHAD.

Особенностью рассматриваемой далее конфигурации core-shell является существование двух симметричных состояний, отличающиеся размещением сосуществующих фаз по core- и shell-положениям [16]. Перестановка фаз меняет площадь межфазной поверхности $A_{c}$ и поверхностную энергию $\sigma_{s}, \sigma_{c s}$, на внешней (shell) и внутренней (core-shell) границах. Это приводит к изменению общей поверхностной энергии (5) и условий фазового равновесия. Поэтому равновесные состояния, соответствующие разным конфигурациям, имеют разный состав и термодинамическую устойчивость сосуществующих фаз.

В качестве объекта моделирования выбран рассмотренный в [18] твердый раствор $\mathrm{Bi}-\mathrm{Sb}$, имеющий верхнюю критическую температуру растворения (ВКТР) при $T=340 \mathrm{~K}$. Функция Гиббса данной системы имеет вид [25]:

$$
\begin{aligned}
& G(x, T)=(6500-2.6 T) x(1-x) \\
& \quad+R T(x \ln x+(1-x) \ln (1-x)) \\
& \quad-R(2.458(1-x)(544.6-T)+2.645 x(903.7-T)),
\end{aligned}
$$

где $x$ - мольная доля $\mathrm{Sb}, R-$ универсальная газовая постоянная.

Одной из проблем подобных вычислений является надежность теплофизических данных о поверхностной энергии веществ в твердом состоянии и межфазных границ. Величина $\sigma_{c s}$ вычислялась как $\sigma_{c s}=0.5\left(\sigma\left(x_{c}\right)+\sigma\left(x_{s}\right)\right)$, подобное приближение использовано в [26]. Для вычисления $\sigma(x)$ использована линейная аппроксимация $\sigma(x)=\sigma_{\mathrm{Bi}}(1-x)+\sigma_{\mathrm{Sb}} x$, где $\sigma_{\mathrm{SB}}=0.350 \mathrm{~J} / \mathrm{m}^{2}$ и $\sigma_{\mathrm{Bi}}=0.521 \mathrm{~J} / \mathrm{m}^{2}$ - поверхностные энергии чистых компонентов, мольные объемы компонентов равны $V_{\mathrm{Sb}}=18.4 \mathrm{~cm}^{3} / \mathrm{mol}$ и $V_{\mathrm{Bi}}=21.3 \mathrm{~cm}^{3} / \mathrm{mol}$ [27].

Функция Гиббса (4) эквимолярной $(x=0.5)$ смеси в координатах $\theta_{1}, \theta_{2}$, для core-shell системы со сферической симметрией радиусом $200 \mathrm{~nm}$ показана на рис. 1 .

Пунктирами на рис. 1, $a$ отмечены „овражные“ траектории, удовлетворяющие условиям $\left(\partial G / \partial \theta_{i}\right)_{\theta_{j}}=0$. Они проходят через гомогенное состояние $G(0,0)$ и локальные минимумы функции Гиббса, соответствующие равновесным гетерогенным состояниям. Рис. 1, $b$ демонстрирует изменение функции Гиббса вдоль этих траекторий. Система, показанная на рис. $1, a, b$ имеет три устойчивых состояния. Глобальному минимуму соответствует стабильное гетерогенное состояние, при котором в core-фазе преобладает Вi. Такой результат 
$a$

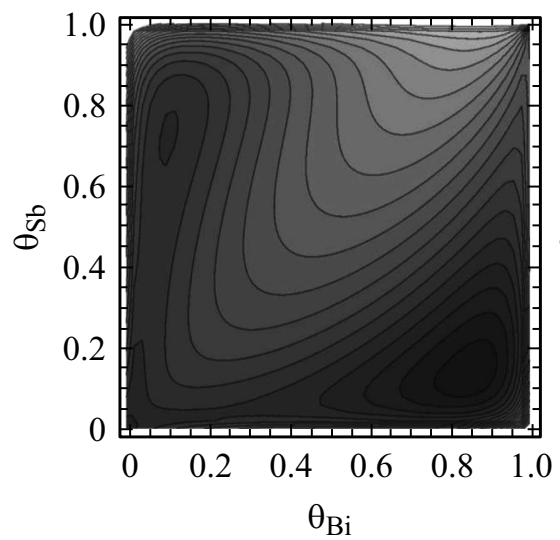

$b$

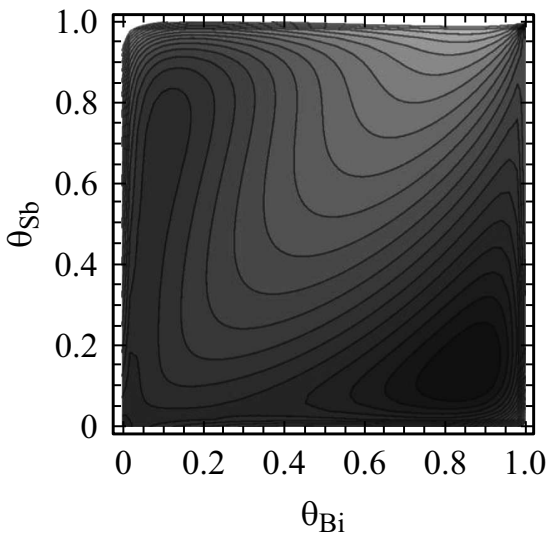

C

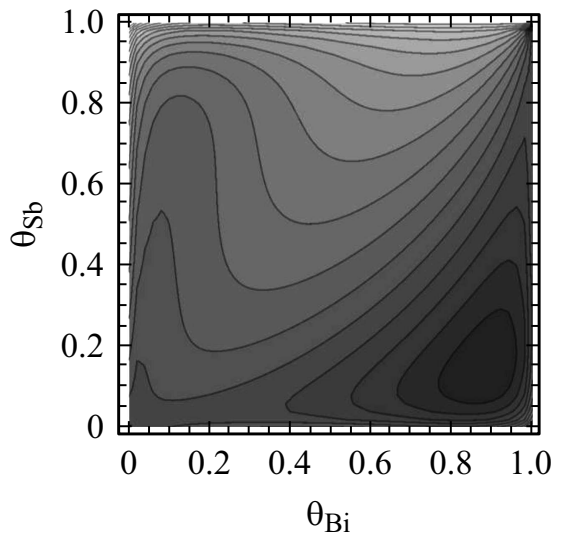

Рис. 2. Функция Гиббса для core-shell частицы $\left(r_{0}=200 \mathrm{~nm}, T=280 \mathrm{~K}, x=0.5\right)$ со сферическим ядром $\left(k_{c}=1\right)$ и несферической оболочкой: $a-k_{s}=1.25, b-k_{s}=1.5$ и $c-k_{s}=3$. Минимумам соответствует более темная окраска.

$a$

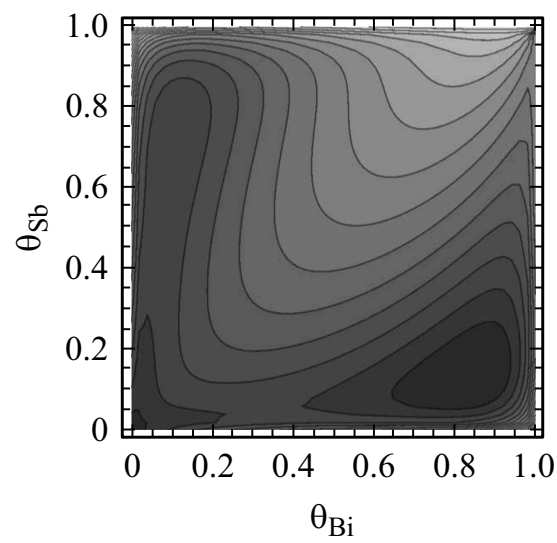

$b$

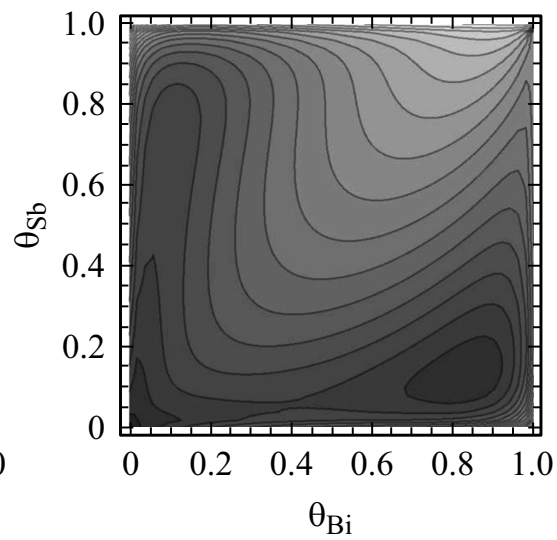

C

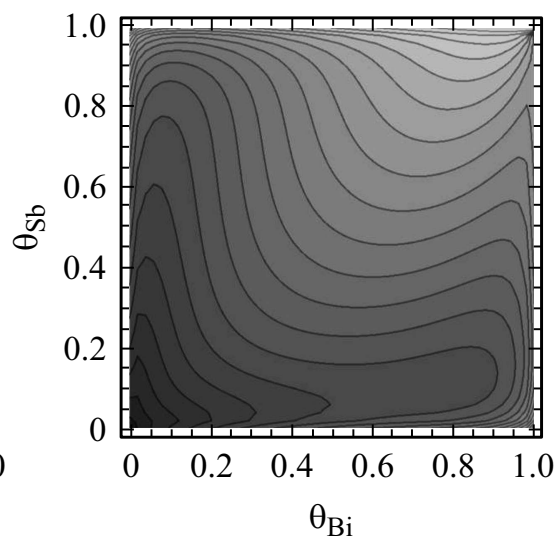

Рис. 3. Функция Гиббса для core-shell частицы $\left(r_{0}=200 \mathrm{~nm}, T=280 \mathrm{~K}, x=0.5\right)$ со сферической оболочкой $\left(k_{s}=1\right)$ и несферическим ядром: $a-k_{c}=1.25, b-k_{c}=1.5$ и $c-k_{c}=3$. Минимумам соответствует более темная окраска.

вполне предсказуем, так как соre-фазу в этом случае образует компонент, у которого больше поверхностная энергия. Гомогенное состояние $G(0,0)$ и гетерогенное состояние, при котором в соге-фазе преобладает $\mathrm{Sb}$, являются метастабильными. При увеличении объема системы гетерогенные состояния вырождаются (имеют одинаковую энергию и фазовый состав), а гомогенное состояние становится термодинамически неустойчивым.

Максимумы на рис. $1, b$, которые отделяют гетерогенные и гомогенное состояния, являются седловыми точками, которые можно отождествить с зародышами новой фазы критического размера, а высоту барьера рассматривать как энергию образования критического зародыша. Существенным отличием от классического определения критического зародыша является то, что система имеет возможность обойти эти состояния по менее выгодным траекториям.

Анализ функции Гиббса, позволяет исследовать закономерности влияния формы внешней и внутренней межфазных границ на фазовые превращения. Перейдем к рассмотрению этого эффекта.

\section{3. Эфффект формы при фазовых превращениях в твердом растворе $\mathrm{Bi}-\mathrm{Sb}$}

Рассмотрим фазовые превращения в системе, имеющей постоянный объем и состав, и разную форму. Форму внешней или внутренней межфазной границы определяют соответствующие параметры $k_{f}$.

Эталоном для сравнения является система со сферической конфигурацией, приведенная на рис. 1. Реакцию этой системы на изменение формы shell- или core-фаз демонстрирует поведение функции Гиббса при отклонении формы внешней или внутренней границ core-shell частицы от сферической (рис. 2-3).

Для демонстрации эффекта формы выбрано несколько значений $k_{f}=1.25,1.5$ и 3 , которые сопоставимы по 

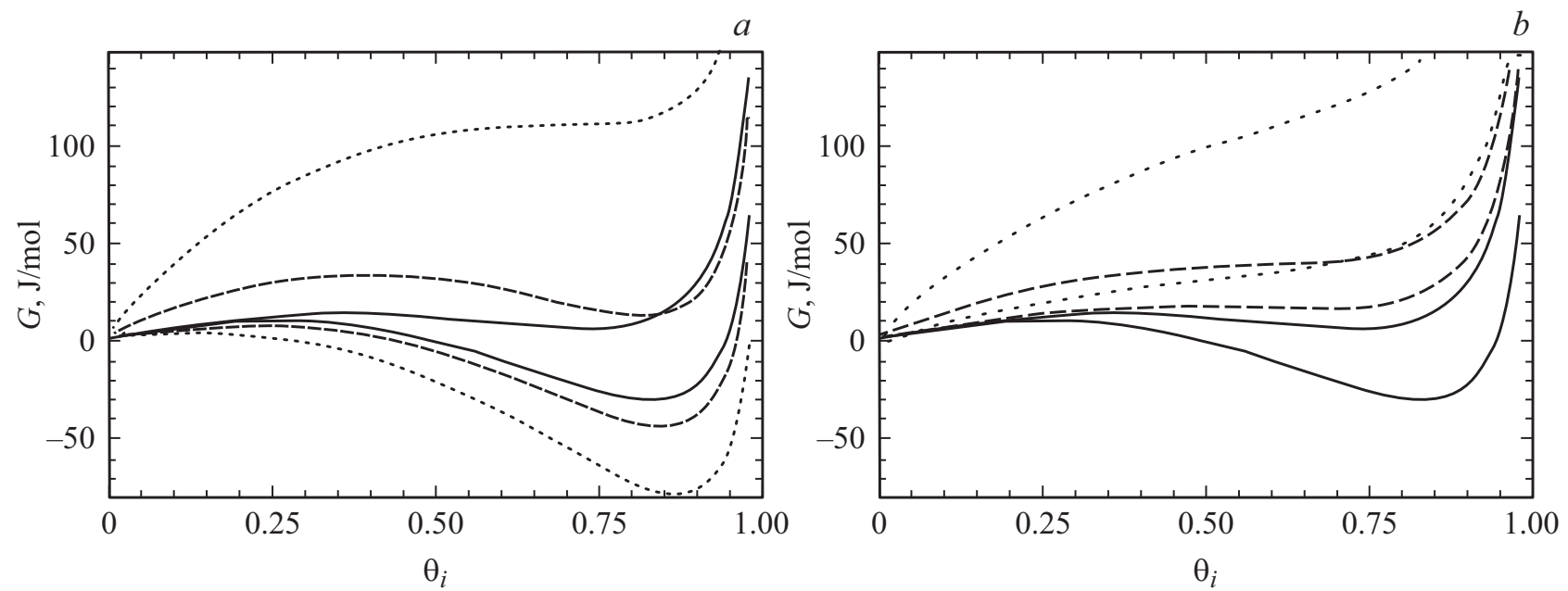

Рис. 4. Изменение функции Гиббса вдоль „овражных“ траекторий (рис. $1, a, b)$ для core-shell частиц $\left(r_{0}=200 \mathrm{~nm}, T=280 \mathrm{~K}\right.$, $x=0.5)$ при $a$ - деформации оболочки, $b-$ деформации ядра. Для $k_{s}=1.5$ и $k_{c}=1.5-$ штриховые линии; для $k_{s}=3$ и $k_{c}=3-$ пунктир. Сплошная линия соответствует эталонной сферической конфигурации $\left(k_{s}=k_{c}=1\right)$.

величине с $k_{f}$ для куба, тетраэдра и прямоугольной пластины с отношением сторон 1:1:0.06 соответственно. Большая часть многогранников, описанных в $[9,14,15]$, имеет $k_{f}<1.2$.

Согласно рис. 2,3 заметное влияние на функцию Гиббса $G\left(\theta_{\mathrm{Bi}}, \theta_{\mathrm{Sb}}\right)$ и число равновесных состояний оказывают и изменение формы оболочки (рис. 2), и изменение формы ядра. Общей закономерностью является исчезновение метастабильного гетерогенного состояния при увеличении $k_{f}$ (деформации) и оболочки, и ядра. Поведение стабильного гетерогенного состояния (глобального минимума) и гомогенного состояния $G(0,0)$ при деформации оболочки и ядра отличаются. При деформации оболочки частицы (с ростом $k_{s}$ ) гомогенное состояние становится неустойчивым. При деформации ядра (с ростом $k_{c}$ ) единственным термодинамически устойчивым состоянием остается гомогенное - распад раствора термодинамически не выгоден. Напротив, стабильное гетерогенное состояние становится единственным термодинамически устойчивым состоянием при больших $k_{s}$, но теряет устойчивость при больших $k_{c}$.

О термодинамической стабильности равновесных состояний можно судить по глубине минимума функции Гиббса и высоте энергетического барьера, отделяющего гомогенное и гетерогенные состояния, которые показаны на рис. 4.

На рис. 4 присутствуют состояния, соответствующие стабильным, метастабильным и безразличным термодинамическим равновесиям и переходы между ними, вызванные преобразованием формы.

На изменение взаимной растворимости компонентов указывает смещение положения минимумов (рис. 2-3). Реакцию состава сосуществующих твердых растворов на изменение формы shell- или core-фаз можно получить при анализе минимумов функции Гиббса, на основе которого построены фазовые диаграммы для сферической и несферических конфигураций с $k_{s}=1.5$ и $k_{c}=1.5$ (рис. $5, a-c)$.

Диаграммы растворимости (рис. 5) демонстрируют влияние формы на фазовые равновесия. Согласно им изменение формы любой межфазной поверхности coreshell наночастиц сопровождается смещением ВКТР и изменением взаимной растворимости компонентов. На диаграммах сплошная линия соответствует кривой растворимости для макроскопической системы. Штриховые кривые соответствуют бинодалям для наночастиц эквимолярного твердого раствора c core-shell конфигурацией. Бинодали стабильного состояния достигают более высоких температур, их левая ветвь соответствует составу core-фазы (обогащается Вi), правая — shellфазы. Для метастабильного состояния левая ветвь соответствует составу shell- фазы, правая - core-фазы (обогащается Вi). Горизонтальные сплошные линии со стрелками ограничивают область температур, в которой гетерогенное состояние сохраняет термодинамическую устойчивость. Формально эту температуру можно интерпретировать как ВКТР рассматриваемого гетерогенного состояния. Горизонтальные штриховые линии со стрелками соответствуют условию $G\left(\theta_{1}, \theta_{2}\right)=G(0,0)$, ниже этой температуры гомогенное состояние является метастабильным.

Приведенные модельные расчеты позволяют утверждать, что

1) деформация оболочки (внешних границ системы) способствует распаду твердого раствора и стабилизации гетерогенного состояния, потенциальный барьер, который необходимо преодолеть при зарождении в гомогенном растворе стабильной core-фазы понижается;

2) core-фаза несферической формы является менее стабильной. Иллюстрацией являются наблюдения преобразования (огрубления) дендритных кристаллов в заполненной раствором полости [28] или преобразования кристалла $\mathrm{NaCl}$, наблюдавшиеся в капле раствора [29]; 

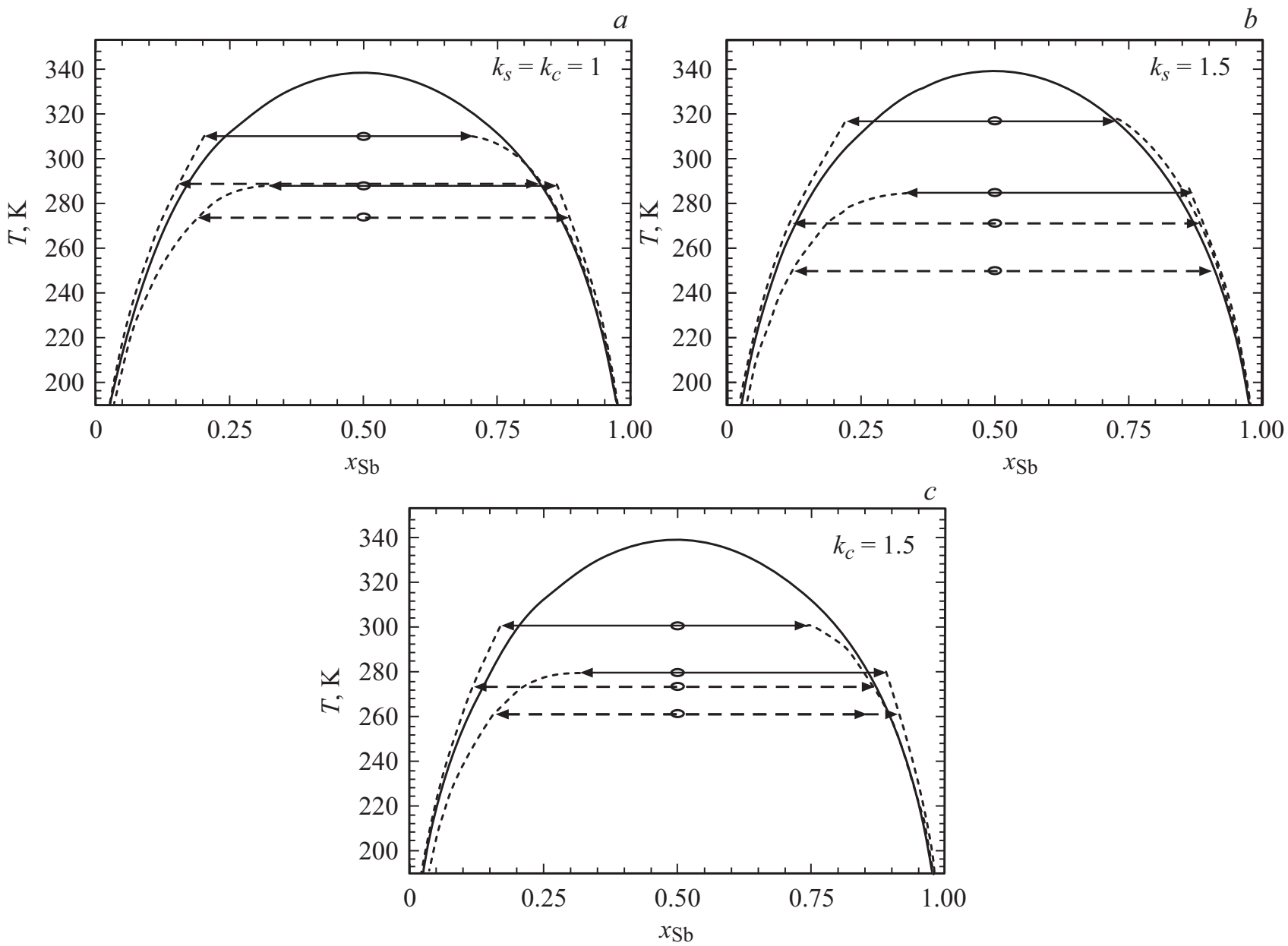

Рис. 5. Диаграммы растворимости для core-shell частиц $\mathrm{Bi}-\mathrm{Sb}\left(r_{0}=200 \mathrm{~nm}, x=0.5\right) a-$ со сферической формой $\left(k_{f}=1\right)$, $b-$ с несферической оболочкой $\left(k_{s}=1.5\right), c-$ с несферическим ядром $\left(k_{c}=1.5\right)$.

3) большие деформации наночастиц позволяют избавиться от возникновения метастабильных core-shell состояний;

4) деформация наночастиц с конфигурацией core-shell приводит к изменению взаимной растворимости компонентов в сосуществующих фазах.

Согласно оценкам образование гетерогенного состояния с оболочкой несферической формы оказалось более выгодным (рис. $4, a$ ), несмотря на увеличение поверхности внешней границы. Следствием может быть самопроизвольное искажение сферической формы. Образование core-фазы несферической формы не выгодно, в этом случае гетерогенное состояние теряет устойчивость при меньших отклонениях от сферической формы. Тем не менее, в достаточно большом интервале условий и это состояние может существовать в метастабильном или безразличном равновесии.

Модельные расчеты позволяют показать, что описанные закономерности очень существенно зависят от соотношения поверхностной энергии и мольных объемов компонентов. В этом можно убедиться, если при использовании той же модели реального раствора (7) и условий $(V, T, x)$ допустить, что $\sigma_{1}=\sigma_{2}$ или $V_{1}=V_{2}$.
Эти же оценки позволяют сделать предположение, что стабилизация гетерогенного состояния связана с переносом компонента с большой поверхностной энергией в core-фазу, это компенсирует увеличение поверхности.

\section{4. Заключение}

Особенности фазовых превращений в системах малого объема не ограничиваются размерной зависимостью диаграмм состояния. Учет эффекта формы дает более общую картину качественных и количественных изменений характеристик фазовых равновесий в наночастицах. В общем случае учет формы межфазных границ не требует существенной модификации расчетных моделей CALPHAD. Как показано в работе его можно свести к использованию множителей $k_{f}(6)$ при расчете поверхностной энергии.

В случае расслаивающихся растворов форма межфазных границ определяет взаимную растворимость компонентов и интервал температур, в котором термодинамически устойчивы гетерогенные состояния. Существенное расширение области метастабильности гетеро- 
генного состояния предполагает возможность получения непрерывного ряда твердых растворов при температурах существенно ниже ВКТР.

Приведенные оценки позволяют утверждать, что деформация гомогенной сферической наночастицы способствует распаду твердого раствора за счет роста термодинамической устойчивости конфигурации coreshell и понижения энергии образования критического зародыша. Отклонение формы core-фазы от сферической менее выгодно и приводит к исчезновению гетерогенного состояния, тем не менее, термодинамическая устойчивость структуры core-shell с несферическим ядром в виде метастабильного состояния сохраняется при достаточно больших деформациях core-фазы.

В общем случае, характер зависимости фазовых превращений от формы в значительной степени определяется соотношением поверхностных энергий и мольных объемов компонентов системы малого объема.

\section{Список литературы}

[1] Ю.И. Петров. Физика малых частиц. Наука, М. (2015). $360 \mathrm{c}$.

[2] L.H. Liang, D. Liu, Q. Jiang. Nanotechnology 14, 4, 438 (2003).

[3] Y. Dahan, G. Makov, R.Z. Shneck. CALPHAD 53, 136 (2016).

[4] A. Roshanghias, J. Vřešt’ál, A. Yakymovych, K.W. Richter, H. Ipser. CALPHAD 49, 101 (2015).

[5] J. Lee, K.J. Sim. CALPHAD 44, 129 (2014).

[6] Y. Eichhammer, M. Heyns, N. Moelans. CALPHAD 35, 2, 173 (2011).

[7] M. Ghasemi, Z. Zanolli, M. Stankovski, J. Johansson. Nanoscale 7, 41, 17387 (2015).

[8] G. Garzel, J. Janczak-Rusch, L. Zabdyr. CALPHAD 36, 52 (2012).

[9] L.D. Marks, L. Peng. J. Phys. Condens. Matter 28, 5, 53001 (2016).

[10] S. Bajaj, M. Haverty, R. Arróyave, W.A. Goddard, S. Shankar. Nanoscale 7, 21, 9868 (2015).

[11] A.S. Shirinyan, M. Wautelet, Y. Belogorodsky. J. Phys. Condens. Matter 18, 8, 2537 (2006).

[12] В.Б. Федосеев, А.В. Шишулин, Е.К. Титаева, Е.Н. Федосеева. ФТТ 58, 10, 2020 (2016).

[13] E.A. Sutter, P.W. Sutter. ACS Nano 4, 8, 4943 (2010).

[14] G. Guisbiers, R. Mendoza-Pérez, L. Bazán-Díaz, R. MendozaCruz, J.J. Velázquez-Salazar, M.J. Yakamán. J. Phys. Chem. C 121, 12, 6930 (2017).

[15] G. Guisbiers. S. Mejía-Rosales, S. Khanal, F, Ruiz-Zapeda, R.L. Whetten, M.J. Yakamán. Nano Lett., 14, 11, 6718 (2014).

[16] В.Б. Федосеев. ФТТ 57, 3, 585 (2015).

[17] В.Б. Федосеев, Е.Н. Федосеева. Письма в ЖЭТФ 97, 7, 473 (2013).

[18] В.Б. Федосеев, Е.Н. Федосеева. ЖФХ 88, 3, 446 (2014).

[19] J. Park, J. Lee. CALPHAD 32, 1, 135 (2008).

[20] T. Tanaka, S. Hara. Zeitschrift fur Met. 92, 11, 1236 (2001).

[21] D. Cholakova, N.D. Denkov, S. Tcholakova, I. Lesov, S.K. Smoukov. Adv. Colloid Int. Sci. 235, 90 (2016).

[22] A.H. Gröschel, A.H.E. Müller. Nanoscale 7, 28, 11841 (2015).

[23] М.Н. Магомедов. ЖТФ 86, 5, 84 (2016).
[24] V.B. Fedoseev, A.A. Potapov, A.V. Shishulin, E.N. Fedoseeva. Eur. Phys. Tech. J. 14, 1, 18 (2017).

[25] М.А. Быков, Г.Ф. Воронин, Н.М. Мухамеджанова. В сб.: Прямые и обратные задачи химической термодинамики. Наука, Новосибирск (1987). С. 30.

[26] D. Hourlier, P. Perrot. Mater. Sci. Forum 653, 77 (2010).

[27] Физические величины. Справочник / Под ред. И.С. Григорьева, Е.3. Мейлихова. Энергоатомиздат, М. (1991). $1232 \mathrm{c}$.

[28] М.О. Клия. Кристаллография 1, 5, 577 (1956).

[29] В.Б. Федосеев. Нелинейная динамика 13, 2, 195 (2017).

Редактор Т.Н. Василевская 